\title{
Study on the Influence of Magnification of Rock SEM on Fractal Dimension
}

\author{
Sen Li \\ College of Civil Engineering and \\ Architecture \\ Shandong University of Science \\ and Technology \\ Qingdao, China \\ E-mail:415060269@qq.com
}

\author{
Houli $\mathrm{Fu}^{*}$ \\ College of Civil Engineering and \\ Architecture \\ Linyi University \\ Linyi, China \\ E-mail:fuhouli6619@sina.com
}

\author{
Zhe Qin \\ College of Civil Engineering and \\ Architecture \\ Shandong University of Science \\ and Technology \\ Qingdao, China \\ E-mail:52349823@qq.com
}

\author{
Lunan Sun \\ College of Civil Engineering and \\ Architecture \\ Shandong University of Science \\ and Technology \\ Qingdao, China \\ E-mail:709858427@qq.com
}

\begin{abstract}
Fractal dimension is widely used in the quantitative description of nonlinear systems and irregular properties. It is of great significance on the mechanism of rock mechanics. This can provide guidance for the management of goafs in rock mines. In this paper, firstly, multiple sets of rock fracture surface images with different magnifications are obtained through SEM experiment. Secondly, the optimal binarization thresholds of each group of images are solved by Otsu method, and the average value is used as the binarization threshold. Finally, the results of fractal dimension is implemented by box-counting method using MATLAB, and the fractal dimension of each group of images under the same threshold is obtained. The results of comparative analysis show that the increase of magnification makes the decrease of observation range. When the structural complexity of the rock surface decreasing, the fractal dimension is decreased. When the magnification is greater than $\mathbf{2 5 0 0}$, the fractal dimension tends to be stable and can be used as a reference for rock fractal research.
\end{abstract}

Keywords - SEM, fractal dimension, box-counting method, MATLAB, magnification

\section{INTRODUCTION}

Fractal geometry is used to describe irregular and chaotic phenomena and behaviors in nature. Mandelbort ${ }^{[1]}$ proposed the concept of fractal dimension and fractal geometry. Once the concept of fractal is presented, it shows its superiority in dealing with extremely irregular traits. The behaviors difficult to describe in traditional geometry can be described by means of fractal geometry. Fractal dimension is the main concept of fractal geometry. It is of great significance to quantitatively describe the non-smooth and irregular properties of nonlinear systems that were difficult to describe in the past. There are a lot of nonlinear systems in nature. Therefore, the fractal theory has been widely used ${ }^{[2]}$.

The structure of rock is relatively continuous in macroscopic view, while the microscopic view is a geometric body composed of particles and voids. It is a typical non-linear body. These cracks are often small and difficult to observe. If you can observe clearly and accurately the enlarged rock image, you can reveal the mechanism of rock mechanics. In the 1980s, SEM was introduced to observe rocks, and the fracture features and morphological characteristics of rock fracture surfaces were obtained, which is very useful for studying the microstructural features of rocks. Many researchers have studied the characteristics of subtle rock under the fractal theory to obtain more results. Zhu Zhende ${ }^{[3]}$ and other people conducted triaxial compression tests on marble under conditions of high water pressure, high surrounding rocks, and low confining pressure, and conducted electron microscopy scans of the fractures to obtain the microscopic characteristics of the rock under this condition; Zuo Jing ${ }^{[4]}$ and other people studied the fractal characteristics of two kinds of rocks by SEM experiment, and proposed the factors that influence the fractal dimension and gap index. It is believed that the magnification and the image threshold have an important influence on the fractal dimension; Wang Fenge ${ }^{[5]}$ used MATLAB to calculate the fractal dimension of the image of microscopic rock and proposed the damage and change law of rock damage.

\section{Digital IMAGe Fractal DimENSION}

\section{A. Calculation method of fractal dimension}

An extremely important concept in fractal dimension theory is the quantitative description of irregular nonlinearity. For rocks, the fractal dimension expresses the roughness of the rock surface and the complexity of cracks in the microcrack. It accurately expresses the small differences in human visual observation and can be used as a measure of comparative research ${ }^{[6]}$. At present, the methods commonly used to find fractal dimensions in various disciplines include_Gray-scale Interpolation, Fractal Brownian Self-similarity Simulation, Differential Box Dimension, and Carpet Overlay ${ }^{[7]}$. The box-counting method is easy to determine the fractal dimension and is convenient for program design. This paper uses the box-counting method to calculate the fractal dimension of the image.

The basic steps of the box-counting method are as follows:

1) Binarization processing the image. The image obtained by the SEM is a grayscale image, that is, each pixel is a number between $0-255$ and the numbers are represented as different grayscales. Select the appropriate threshold value and convert the grayscale image into a binary image. There are only two colors_-Black and White, namely, 0 for white and 1 for black. This translates the image into a matrix of only 0 and 1 , and each pixel is represented by a number.

2) Take a box with a side length of $K$ to divide the image matrix, and the partitioned areas do not overlap; Record the number of areas containing black (white) parts, denoted as 
$N(k)$; Usually taken $k=1,2,4, \cdots, 2^{i}(i=0,1,2,3, \cdots)$; From the image, the pixel size $1,2,4, \cdots, 2^{i}$ are divides the image by the length of the edge and finally gets the number of boxes $N(1), N(2), N(4), \cdots, N\left(2^{i}\right)$. When the $k$ is tending to 0 , the fractal dimension of box dimension method is as in (1):

$$
D=\lim _{k \rightarrow 0} \frac{\lg N(k)}{\lg k}
$$

3) It is known from (1) that the smaller the $\mathrm{k}$ is, the more accurate the fractal dimension calculation result is. However, for the image, $\mathrm{k}$ has the minimum value as a single pixel, and the fractal dimension can be obtained by fitting a straight line. Fitting the $N(k)$ in logarithmic coordinates corresponding to the different size $\mathrm{k}$ gives the following formula:

$$
\lg N(k)=D^{*} \lg k+h
$$

The slope of the straight line is the fractal dimension.

\section{B. MATLAB implementation of the box dimension method}

MATLAB has powerful matrix operations, numerical analysis, and graphics processing functions, and it can be conveniently programmed. In particular, its digital image processing technology can be used to program the box dimension method ${ }^{[8]}$. The SEM result is a grayscale image. Due to a variety of factors in the image acquisition, the image brightness is uneven and the image quality is poor. Therefore, the image should be preprocessed first mainly including image enhancement and image denoising. The process can be easily implemented by MATLAB ${ }^{[9]}$. After the image is binarized, the image file is converted into a matrix of 0 and 1 , and then the analysis dimension is calculated. This paper uses MATLAB programming to implement the box dimension method.

\section{SEM EXPERIMENT}

\section{A. Experimental facilities}

Electron microscopy is widely used in the surface characterization of nano-scale samples of metallic materials, polymer materials, and inorganic materials. It is the most effective instrument for observing the morphology of materials. The electron microscope type used in this experiment was produced by FEI, USA, and the model was Nova Nano SEM450. The specific parameters are as follows:

High vacuum mode resolution: $1 \mathrm{~nm}(15 \mathrm{KV}) ; 1.6 \mathrm{~nm}$ $(1 \mathrm{KV})$;

Low vacuum mode resolution: $1.5 \mathrm{~nm}$ (10KV, Helix detector), $1.8 \mathrm{~nm}$ ( $3 \mathrm{KV}$ Helix detector);

Sample magnification: 40 to 400,000 ;

Movement range of sample table: $\mathrm{X}=\mathrm{Y}=110 \mathrm{~mm}$;

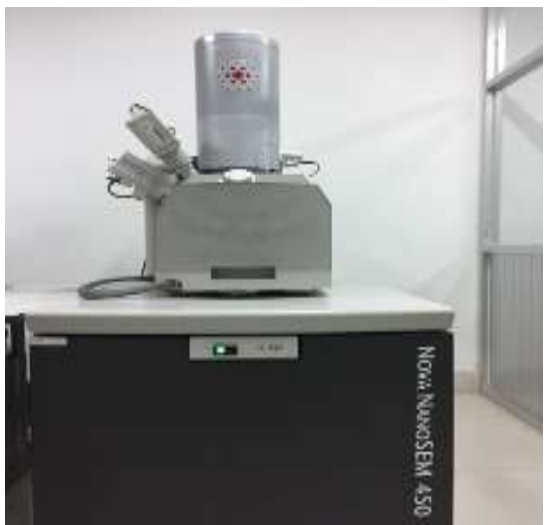

Fig. 1. High resolution SEM

he device has an ultra-high resolution, high-performance $\mathrm{X}$-ray energy spectrometer; capable of qualitative, semiquantitative and quantitative analysis of the sample; with a comprehensive analysis of the morphology and chemical composition.

\section{B. Experimental process}

The rock samples used in the experiment were all collected from the side slope of a gold mine. The main component of the rock sample was beresitization granite. Rocks in the area have been affected by the natural environment for ling time, and the surface has weathered and cracked. In the laboratory sampling, the rock was first polished and dried, and a core with a diameter of $5 \mathrm{~cm}$ was drilled with a rock core machine to make a core sample with a thickness of $1 \mathrm{~cm}$. External forces are applied to the rock chip to make it fracture and failure. The appropriate rock sample is taken as the object of the study and the fracture surface is observed. The rock sample was placed in the coating film inside the coating machine, and then SEM experiments were conducted. A total of 20 sets of images at different positions were collected, and the magnifications were 500, 800, 1500, 2500, and 3500. Figure 2 is an image of the same position with a magnification of 800 (a) and $1500(b)$.
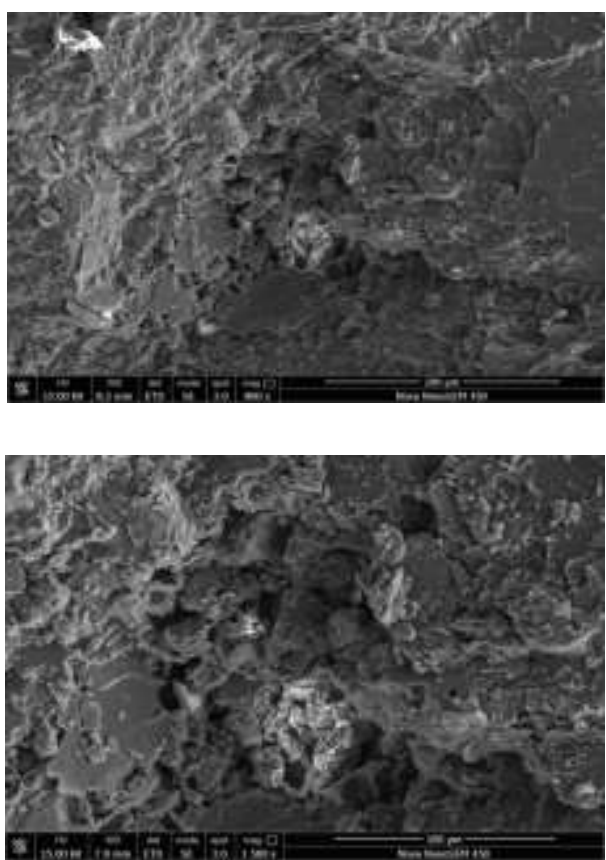

Fig. 2. SEM images 
From the SEM images, it was found that the fracture surface of the granite was squamous, and the surface was extremely uneven and the pores were developed. The broken pieces were mostly schistose and squamous. By observing the image directly, it is difficult to quantitatively characterize the surface of the rock.

\section{ANALYSIS OF EXPERIMENTAL RESULTS}

The threshold of binary image exerts important influence on calculation of fractal dimension, therefore we must ensure that the threshold of binary image is the same when conducting research on the impact of magnification. However, the best binarization thresholds are different for diverse images, hence the essay applies the method of $\mathrm{Otsu}^{[10]}$ to solve the best thresholds of different image binarizations, and calculate the average value of the best thresholds of different image binarizations with different magnifications in the same position, as the uniform binarization threshold. Typical images of Group 1, 3, 4, and 5 are selected as the subjects of study, with magnifications of $500,800,1500$, and 3500 . Table 1 is the best binarization thresholds by the method of Otsu .

TABLE I. RESULTS OF BEST BINARIZATION THRESHOLDS WITH DIFFERENT MAGNIFICATIONS

\begin{tabular}{ccccc}
\hline & 1 & 3 & 4 & 5 \\
\hline 500 & 0.353 & 0.416 & 0.341 & 0.447 \\
800 & 0.341 & 0.404 & 0.345 & 0.443 \\
1500 & 0.322 & 0.424 & 0.357 & 0.420 \\
2500 & 0.353 & 0.418 & 0.359 & 0.458 \\
3500 & 0.353 & 0.410 & 0.368 & 0.467 \\
Average & 0.344 & 0.414 & 0.354 & 0.447 \\
\hline
\end{tabular}

The results in table 1 shows that the best binarization thresholds in different positions of rock samples are different, while the best binarization thresholds change a little in the same position of rock samples with different magnifications, therefore the average value could serve as the uniform binarization threshold. The box-counting method could be implemented by MATLAB and the number of boxes in the images $N(k)$ could be calculated by the average value of the best binarization thresholds. The number of boxes $N(k)$ corresponds to different sizes $k$, which is the fitting line. The absolute value of the fitting curve slope is the fractal dimension of the corresponding images. Figure 3 is the results of fitting line with magnification of 800 of each serial number.

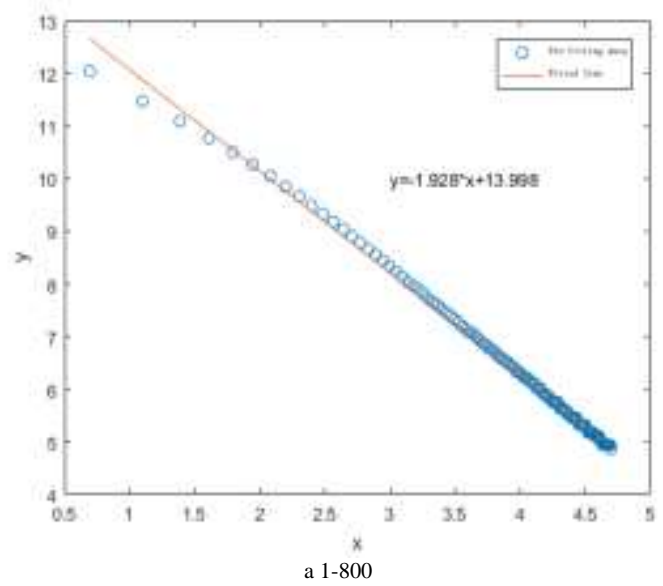

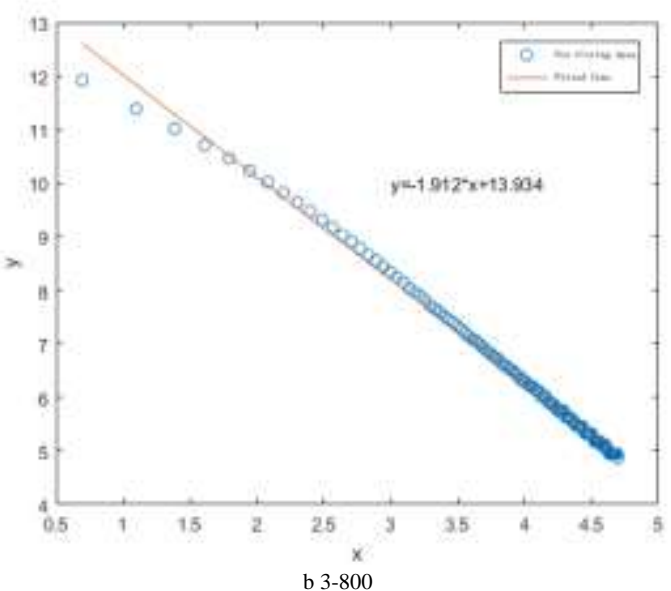
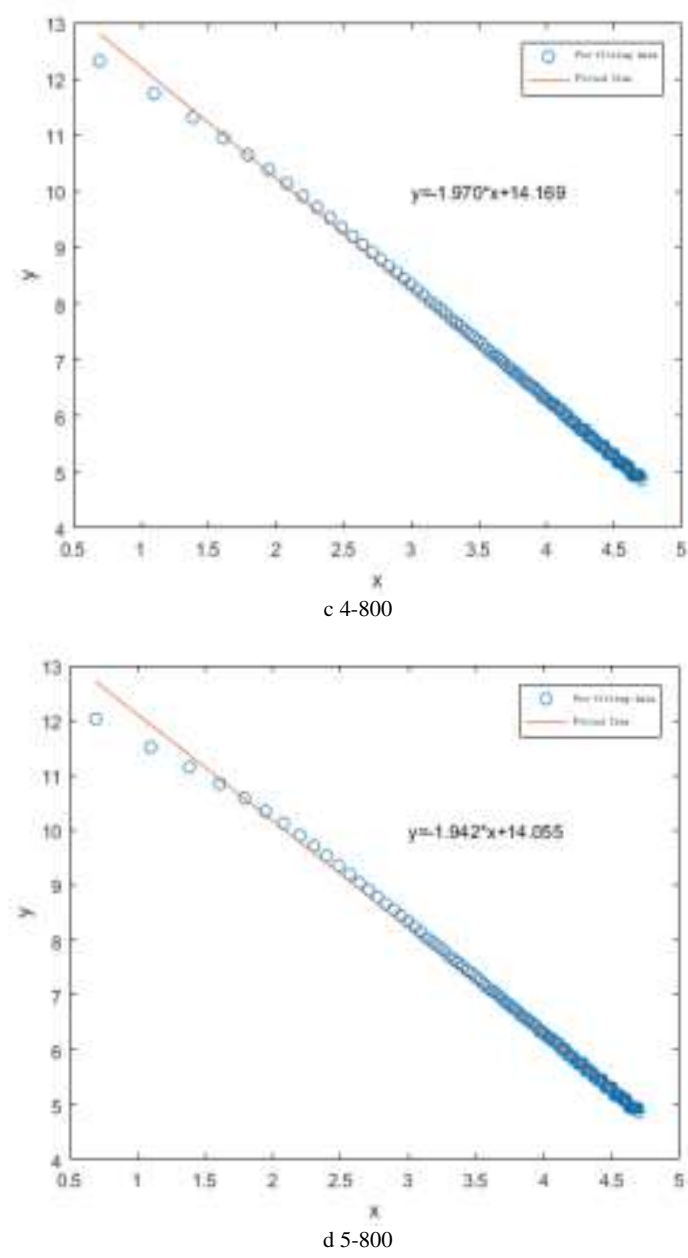

Fig. 3. The results of fitting line with magnification of 800

(Note: Serial number 1-800 is the image of the first group with magnification of 800 , and the like.)

The fitting results possess good linearity, which indicates that the the fractal dimension with the box-counting method has comparatively high precision. Figure 4 shows the results of fractal dimension of the coding images with different magnifications calculated with the method above. According to the curve in the picture, the magnification has large impact on the fractal dimension and the fractal dimension nonlinear decreases with the enlargement of magnification. When the magnification is small, the amplitude of fractal dimension decreasing increases. When the magnification is 
greater than 2500, the fractal dimension tends to be stable. The main reason is that with the increase of magnification, the range of observation decreases. Although we could observe more detailed rock structure, the complexity of structure change on rock surface decreases and the fractal dimension decreases. Therefore we should avoid selecting images with low magnification when studying the fractal dimension of rock images. Those with high magnification are more stable.

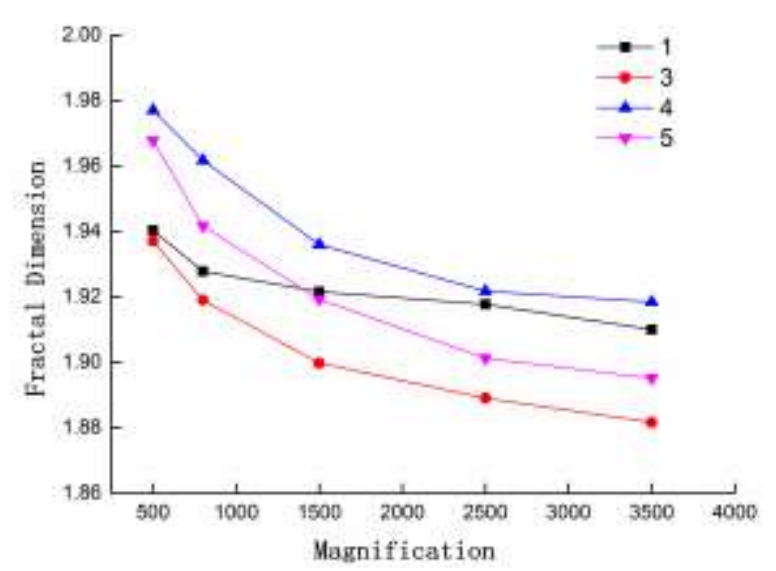

Fig. 4. Fractal dimension curve with different magnifications

\section{CONCLUSION}

The essay focuses on study the influence of image magnification on fractal dimension though the SEM experiment of granite. When the binarization threshold is same in the same position of rock samples, the results shows that the fractal dimension decreases with the increase of rock samples magnification. The fundamental reason is the increase of magnification leads to the decrease of the rock surface complexity and the fractal dimension. When the magnification is small, the fractal dimension could not exactly reflect the structure features of the rock sample.
When the magnification is greater than 2500, the fractal dimension tends to be stable, the result is more valuable for reference.

\section{ACKNOWLEDGEMENT}

In this paper, the research is supported by the Shandong Provincial Natural Science Foundation (Project ZR2017BEE014) and Scientific Research Foundation of Shandong University of Science and Technology for Recruited Talents (Project 2017RCJJ050).

\section{REFERENCES}

[1] MANDELBROT B B. Stochastic models for the Earth's rclicf,the shape and the fractal dimension of the coastlines, and the number-area rule for islands[J]. Proceedings of the National Academy of Sciences, 1975,72(10):3825-3828.

[2] Peng Ruidong, Xie Heping, Ju Yang. Computation Method of Fractal Dimension for 2-D Digital Image[J]. Journal of China University of Mining \& Technology, 2004, 33( 1): 19-25.

[3] Zhu Zhende, Zhang Yong, Xu Weiya, etc. Experimental Studies and Microscopic Mechanics Analysis on Marble Rupture under High Confining Pressure and High Hydraulic Pressure[J]. Chinese Journal of Rock Mechanics and Engineering, 2005, 21(1): 44-51.

[4] Zuo Jing, Xu Weiya, Wang Huailing, etc. Fractal Analysis of SEM Image for Rocks[J].Journal of China Three Gorges University(Natural Sciences), 2014, 36(2): 72-76.

[5] Wang Feng'e , Zhu Changxing. Realization of Damage Fractal Dimensions of Rock SEM Image in the MATLAB Environment[J]. Ship Electronic Engineering, 2009, 29(08): 144-146.

[6] Zhao Haiying, Yang Guangjun , Xu Zhengguang. Comparison of Calculation Methods-Based Image Fractal Dimension[J].Computer Systems Applications, 2010, 20(3): 238-241

[7] Yang Shushen, Shao Longyi. Estimation of Fractal Dimensions of Images Based on MATLAB[J]. Journal of China University of Mining \& Technology, 2006, 35(4): 478-481.

[8] Zhou Pin. Image processing and graphical user interface design on MATLAB[M].Beijing: Tsinghua University Press, 2013.

[9] Gao Zhanhong, Xu Wenbo. Image Processing Cases Studies Based on MATLAB[M].Beijing: Tsinghua University Press, 2011.

[10] Bu Feiyu, Zhu Qing, Wang Tao. An Improved Binarization Method Based on Maximum Variance between Clusters[J].Computer Knowledge and Technology, 2015, 11(05): 188-189+195. 